\title{
Corneal Burn
}

National Cancer Institute

\section{Source}

National Cancer Institute. Corneal Burn. NCI Thesaurus. Code C50506.

Chemical or radiant injury to the cornea. 